\title{
Redes sociales en el marco de la sustentabilidad educativa en tiempos de pandemia
}

\section{Social networks within the framework of educational sustainability in times of pandemic}

\author{
Witre Omar Padilla ${ }^{1 *}$ D, Manuel Emilio Milla Pino ${ }^{2}$ (D), Andre Jorge Renzo Vargas Tello ${ }^{1}$, Zoila Raquel Requejo
} Calderón ${ }^{1}(\mathbb{D})$, Luz Aurora Rios Requejo ${ }^{1}$, Narumi Elizabeth Hidalgo Nisshioka' ${ }^{1}$ (D)

\section{RESUMEN}

Se evaluó el impacto de un programa de orientación educativa por redes sociales sobre tres dimensiones relacionadas con la pandemia COVID-19, en sujetos personas residentes del área urbana de la provincia de Chachapoyas, Perú, durante el periodo septiembre-diciembre 2020. El programa consistió en la difusión por redes sociales de la información científica actualizada sobre etiología, prevención y terapéutica de la enfermedad, y la transferencia de técnicas mentales para proteger la salud emocional, mediante estrategias de comunicación accesibles y amenas. Las dimensiones estudiadas fueron nivel de conocimiento sobre la pandemia, cuidados y medidas preventivas y la gestión de la salud emocional, mediante un instrumento tipo Likert de 12 ítems que se aplicó antes y después de la ejecución del programa. Se compararon los puntajes alcanzados por cada una de las dimensiones, las observadas entre las dimensiones, y también se midió la uniformidad de las opiniones de los sujetos en cada dimensión tanto antes como después. Se encontró un aumento en el puntaje en cada una de las dimensiones después de la aplicación del programa, siendo estadísticamente significativo para la gestión de la salud emocional. Se observó diferencias significativas en los puntajes entre las tres dimensiones tanto antes como después, con mayor fuerza en la de cuidados y medidas preventivas. También el programa produjo uniformidad en las opiniones de los sujetos sobre cada una de las dimensiones. Estos resultados sugieren que la transferencia por redes sociales, de conocimientos y métodos saludables para enseñar a las personas a cuidarse de la COVID-19, es una estrategia viable y potencialmente sustentable.

Palabras clave: redes sociales, sustentabilidad educativa, pandemia COVID-19.

\begin{abstract}
The impact of an educational guidance program through social networks on three dimensions related to the COVID19 pandemic was evaluated, in subjects living in the urban area of the province of Chachapoyas, Peru, during the period September-December 2020. The program consisted of in the dissemination through social networks of up-todate scientific information on the etiology, prevention and therapy of the disease, and the transfer of mental techniques to protect emotional health, through accessible and enjoyable communication strategies. The dimensions studied were level of knowledge about the pandemic, care and preventive measures, and the management of emotional health, using a 12-item Likert-type instrument that was applied before and after the execution of the program. The scores achieved by each of the dimensions were compared, those observed between the dimensions, and the uniformity of the opinions of the subjects in each dimension was also measured both before and after. An increase in the score was found in each of the dimensions after the application of the program, being statistically significant for the management of emotional health. Significant differences were observed in the scores between the three dimensions both before and after, with greater force in that of care and preventive measures. The program also produced uniformity in the opinions of the subjects on each of the dimensions. These results suggest that the transfer of knowledge and healthy methods through social networks to teach people to take care of COVID-19 is a viable and potentially sustainable strategy.
\end{abstract}

Keywords: social networks, educational sustainability, COVID-19 pandemic..

\footnotetext{
${ }^{1}$ Universidad Nacional Toribio Rodríguez de Mendoza de Amazonas, Facultad de Ciencias de la Salud, Chachapoyas, Perú. ${ }^{2}$ Universidad Nacional de Jaén, Facultad de Ingeniería Civil, Jaén, Perú.

*Autor de correspondencia. E-mail: vomarpadilla@gmail.com
} 


\section{INTRODUCCIÓN}

Las redes sociales (Social Networks Sites) son aplicaciones basadas en Internet desarrolladas sobre los fundamentos de la Web 2.0, que permiten la creación y el intercambio de contenidos generados por el usuario en una comunidad virtual, haciendo que las personas con intereses similares se puedan conectar y establecer una relación (Serri, 2018).

En el contexto mundial actual, dominado por la pandemia COVID-19, producida por el coronavirus SARS$\mathrm{CoV}-2$, las redes sociales se han posicionado como los medios más utilizados para llevar a la gente información sobre los distintos aspectos relacionados con la enfermedad y otros temas esenciales en la vida de las personas, como los familiares y laborales (Hao y Basu, 2020), así como también han sido un soporte importante en la educación virtual y el trabajo remoto, que fueron adoptados por la mayoría de los países del mundo, como una respuesta viable para darle continuidad a su existencia como naciones, incluidos sus respectivos sistemas educativos y económicos, contribuyendo con ello a evitar la amenaza del colapso que se presagiaba desde el inicio de la enfermedad (Catalán-Matamoros, 2020; Tala y Vásquez, 2020). Por los momentos, y aun con la llegada de las vacunas contra el coronavirus causante de la COVID-19, no se puede predecir por cuánto tiempo más se mantendrá la emergencia sanitaria y, por lo tanto, se vislumbra que las redes sociales seguirán teniendo un papel relevante en la sociedad.

Una proporción importante de la información difundida por las redes sociales sobre aspectos fundamentales de la enfermedad, necesarios para educar a la población en cuanto a las medidas de protección y tratamiento adecuados, sigue siendo contradictoria y procede de fuentes poco creíbles, formando parte del desorden informativo llamado infodemia que provoca confusión en la sociedad (Acosta-Quiroz e IglesiaOsore, 2020; Salaverria et al., 2020), y contribuye en generar en la gente un abanico de actitudes desproporcionadas frente a la amenaza de la enfermedad, el cual comprende desde una indiferencia extrema que ponen en riesgo su salud y de las demás personas de su entor- no, hasta comportamientos que revelan un desbordamiento del equilibrio emocional y mental como estados de pánico, conductas obsesiva-compulsivas e incluso cuadros psiquiátricos (Buitrago et al., 2020; Sandin et al., 2020).

Las redes sociales como fuentes de información accesibles a muchas personas en diferentes lugares y momentos, bien pudieran ser vías eficientes para transferir y empoderar a las poblaciones de la información científica fidedigna, actualizada y validada por las instituciones rectoras de la salud, para que de manera ecológica aumenten sus niveles de conciencia en cuanto a la causa, epidemiologia, evolución clínica, prevención y tratamiento correctos, contribuyendo así a que actúen de manera adecuada y asuman la responsabilidad de su propia protección ante la pandemia y sus riesgos (Álvarez-Mon et al., 2020; Bohórquez et al., 2020), y de esa manera aprovechar su fuerza de difusión para convertirlas en aliadas en la promoción de una cultura saludable en el contexto de la emergencia sanitaria y en la vida en general, como una estrategia útil en la búsqueda de un desarrollo humano sustentable (Navarro Rodríguez et al., 2020).

En virtud de que no hemos encontrado en la literatura científica revisada, investigaciones que hayan utilizado a estas herramientas para educar a la población sobre la pandemia, nos propusimos en este estudio, desarrollar un programa de orientación educativa en salud por redes sociales, mediante la transferencia de información científica sobre la enfermedad, así como también de tecnologías mentales útiles para el adecuado manejo de las emociones, con el objetivo de mejorar el nivel de conocimiento sobre la pandemia, promover el uso de los recursos y métodos adecuados para la prevención y brindar estrategias provechosas para la gestión de las emociones en este contexto pandémico, en la provincia de Chachapoyas del departamento de Amazonas, una de las localidades más afectadas por la COVID.19 en el Perú, durante el año 2020 (González Castillo et al., 2020; Llerena Lanza y Sánchez Narváez, 2020). 


\section{MATERIALY MÉTODO}

Población, muestra, fecha, criterios de selección y aspectos éticos.

El estudio se realizó en sujetos mayores de 18 años de edad, residentes del área urbana de la provincia de Chachapoyas del departamento de Amazonas, Perú, cuya población está constituida por 11917 hombres y 13615 mujeres (INEI, 2017) durante el periodo septiembre-diciembre de 2020, que utilizan redes sociales y voluntariamente manifestaron su decisión y dieron su consentimiento por escrito para participar en el estudio. El tamaño de la muestra fue determinado mediante procedimiento estadístico, aplicando la fórmula para poblaciones finitas y conocidas (Aguilar-Barojas, 2005), resultando de 53 sujetos.

\section{Criterios de inclusión}

Individuos que, al menos, naveguen dos horas diaria por sus redes sociales de preferencia.

\section{Criterios de exclusión}

Individuos que manifestaron no ser residentes de la provincia del estudio o que tenían dificultades en el acceso a internet.

\section{Diseño de investigación}

Se trata de una investigación pre-experimental, de nivel aplicativo, prospectivo y longitudinal en la que se describen las características demográficas de la población de estudio, incluyendo la edad, el sexo y el nivel de estudio alcanzado, el comportamiento de los ítems (indicadores) antes y después de la aplicación de un programa de orientación educativa sobre la COVID19, y se evaluó el efecto de este programa en tres dimensiones relacionadas con la pandemia.

El estudio se programó para ser ejecutado en dos etapas de ocho semanas cada una. La primera etapa se realizó de septiembre a octubre con 25 participantes y la segunda de noviembre a diciembre con 28 participantes.

Los sujetos eran incorporados en la investigación durante las dos primeras semanas del inicio de cada etapa, mediante el muestro no probabilística bola de nieve. Para ello, los investigadores utilizaron sus redes sociales para enviar a sus contactos una encuesta de 4 preguntas donde se indagaba sobre su red social de preferencia, horas y horario dedicados a navegar en la red y si estarían interesados (as) en participar en un programa de orientación donde recibirían por su red social preferida, información científicamente validada sobre la pandemia COVID-19 y en el cual se le mantendría en privado su identidad. Además, se les solicitaba que difundieran la encuesta a sus contactos mayores de 18 años en sus redes sociales. A cada persona que respondió afirmativamente, de inmediato se le daba acceso a la página web del Proyecto, identificada con el nombre de "Revisión Informativa de la COVID-19" para que respondiera un cuestionario y de esta manera iniciaba su participación en el estudio.

\section{Instrumento de medición.}

El instrumento de medición utilizado fue un cuestionario tipo Likert de 12 ítems, con cinco alternativas de respuesta en escala ordinal para cada una, diseñado por los investigadores. Antes de su aplicación se realizó la validez de contenido a través de juicio de expertos y la confiabilidad se determinó mediante una prueba piloto en línea a 20 individuos con el mismo perfil de la población en estudio y la aplicación de la prueba alfa de Cronbach, resultando ésta de 0,78.

\section{Dimensiones del estudio.}

Tres fueron las dimensiones estudiadas, que se estructuraron de la siguiente manera:

Nivel de conocimiento sobre la pandemia.

Comprende los ítems 1 al 4, en los que se indagaba sobre la causa de la enfermedad (ítem 1), forma de transmisión o contagio más común (ítem 2 ), la muerte como el desenlace más frecuente en la evolución de la enfermedad (ítem 3) y la utilidad del consumo de medicamentos como forma de prevención (ítem 4).

Cuidados y medidas preventivas implementadas en la cotidianidad.

Con los ítems 5 al 8, donde se preguntaba sobre el hábito de lavado de manos con agua y jabón o uso de desinfectante en las últimas 24 horas (ítem 5), uso en la última semana de mascarilla sanitaria estando en lugares públicos o con personas con quienes no convive (ítem 6), contacto directo en las últimas 24 horas con personas con quienes no está conviviendo (ítem 7) y 
consumo en la última semana de medicamentos de farmacia o productos naturales automedicados, para prevenir la COVID-19 (ítem 8).

Gestión o manejo de la salud emocional.

Incluye los ítems 9 al 12, que indagaba sobre la frecuencia de sentimientos de nerviosismo e intranquilidad en la última semana (ítem 9), sentimientos de depresión en la última semana (ítem 10), preocupación en la última semana porque el propio sujeto o un familiar cercano pudiera enfermar gravemente por COVID19 (ítem 11), y experiencias en la última semana de ideas, pensamientos o sentimientos repetidos de que pudiera estar enfermo(a) de COVID-19 (ítem 12).

Para determinar el nivel o fuerza de cada ítem se utilizó la siguiente escala de clasificación: $5=$ Nivel muy alto; $4=$ Nivel alto; $3=$ Nivel medio; $2=$ Nivel bajo; 1 = nivel muy bajo.

Un nivel muy alto (5) se consideró que el sujeto tenía un conocimiento correcto, aplicaba las estrategias de prevención adecuadas y/o gestionaba apropiadamente sus emociones en el contexto de la pandemia. Por el contrario, un nivel muy bajo se estimó que los sujetos carecían de los conocimientos, no aplicaban las estrategias de prevención correctas y/o manejaban inapropiadamente sus emociones.

\section{Diseño del programa de orientación educativa sobre la pandemia COVID-19.}

Para realizar la intervención educativa se diseñó e implementó un programa que fue estructurado teniendo en cuenta las dimensiones de interés antes descritas. Este programa fue elaborado por los autores del proyecto y se desarrolló desde la semana 3 a la semana 8 de su ejecución; y consistió en la difusión por las redes sociales más utilizadas por los sujetos participantes, de información científica actualizada para ese momento, publicada en revistas indizadas, validada y aprobada como cierta por la Organización Mundial de la Salud (OMS) y el Ministerio de Salud del Perú (MINSA). Esta información fue organizada y presentada en cuatro grandes temas relacionados con la enfermedad: 1.Etiología, epidemiologia, manifestaciones clínicas, evolución y tratamiento; 2.- Estrategias y métodos de prevención; 3.- El papel de la medicación y automedicación de productos farmacéuticos y no farmacéuticos en la prevención, y 4.- Estrategias de tecnologías mentales para el manejo y gestión de las emociones y la salud emocional ante la pandemia.

Cuatro de los investigadores se encargaron de conducir este programa en todas sus fases: la primera fase consistió en la selección del material con los contenidos temáticos de interés a partir de las fuentes válidas y creíbles; la segunda fue la elaboración de artículos, videos, audios y entrevistas cortos con esa información realizada por los investigadores, redactados o narrados en lenguaje ameno y llano para promover la atención y facilitar la comprensión por parte de los sujetos. Estos documentos eran revisados, corregidos y aprobados por el investigador principal antes de su publicación.

Aplicación del programa de orientación educativa sobre la pandemia COVID-19.

Cada documento generado durante la fase de diseño era publicado una sola vez por las redes sociales preferidas de los sujetos participantes y también era colgado en la página web del proyecto, accesible de manera permanente tanto a los participantes como a todo público visitante de la misma. Cada día, de lunes a viernes, y durante las seis semanas después del enrolamiento de los sujetos en el proyecto, se publicaba un documento nuevo y se motivaba a los participantes para su revisión. Al final de cada semana, los investigadores solicitaban a los sujetos una retroalimentación sobre el contenido de los documentos presentados y los aprendizajes logrados. Los documentos utilizados en la segunda etapa eran similares a los de la primera, con las actualizaciones pertinentes de acuerdo a los avances científicos en el tema de la pandemia y los criterios emanados de la OMS y el MINSA.

En la octava semana, una vez finalizado el programa de orientación, se les solicitaba a los sujetos participantes que accedieran a la página web del proyecto para responder el cuestionario y, con ello, se daba por finalizado el desarrollo del programa.

\section{Análisis de datos}

Los datos fueron procesados en Excel para obtener la 
puntuación de cada ítem y la puntuación total de cada dimensión, tanto antes como después de la aplicación del programa de orientación. Esta última se realizó mediante la suma de los valores asignados por un mismo sujeto a cada uno de los cuatro ítems de la respectiva dimensión. Se registró el estadístico la moda para determinar el comportamiento de los ítems antes y después.

Con la finalidad de evaluar el impacto del programa de orientación sobre cada una de las tres dimensiones estudiadas, se compararon las puntuaciones totales antes y después para cada dimensión, mediante la prueba estadística de " $\mathrm{t}$ " de Student para muestras relacionadas o pareadas. Además, para determinar la existencia o no de uniformidad entre las opiniones o juicios emitidos por los sujetos y las posibles diferencias entre los puntajes que éstos asignaron a las dimensiones tanto antes como después, se aplicó la prueba de Friedman (Rubio Hurtado y Berlanga Silvente, 2012). Un valor de $\mathrm{P}<0,05$ se consideró que no pertenecían al mismo universo y, por lo tanto, estadísticamente significativo. El análisis estadístico de los datos se realizó con el software Statistix 8.0. Los resultados relevantes se presentan en tablas.

\section{RESULTADOS}

Demografía y redes sociales.

De los cincuenta y tres participantes que iniciaron, cuarenta y ocho se mantuvieron hasta el final del estudio, hombres y mujeres en su gran mayoría en edades comprendidas entre los 18 a 34 años $(72,9 \%)$, con nivel educativo de secundaria, técnica o universitaria $(97,9 \%)$, que utilizan las redes sociales Facebook y/o
WhatsApp (91,6\%), en horario vespertino o nocturno $(87,5 \%)$, durante 2 a 8 horas diarias $(72,9 \%)$.

Comportamiento de los ítems antes y después del programa de orientación educativa.

La mayoría de los ítems lograron puntuaciones altas o muy altas tanto antes como después del programa. La moda de dos ítems (6 y 8) se iniciaron y mantuvieron con niveles muy altos, mientras que las de cinco de ellos $(3,4,7,9$ y 12$)$ se mantuvieron en niveles altos, cuatro $(1,2,5$ y10) subieron de alto a muy alto, y solo un ítem (11) subió de nivel medio a alto.

Dimensionalidad del programa de orientación educativa.

La Tabla 1 muestra los puntajes alcanzados por cada una de las dimensiones estudiadas, antes y después de la aplicación del programa de orientación. La mínima puntuación que podía lograr cada dimensión era de 192, y la máxima de 960. En cuanto a las tres dimensiones, se observa en la tabla 1, que cada una presenta puntuaciones altas antes, con un aumento consistente en cada una de ellas después, siendo este aumento altamente significativo $(\mathrm{P}=0,0017)$ en la dimensión gestión de la salud emocional. También se observa que la dimensión con la mayor puntuación antes y después fue la de cuidados y medidas preventivas.

Uniformidad entre las opiniones o juicios emitidos.

La Tabla 2 muestra los resultados estadísticos de la uniformidad o no entre las opiniones o juicios emitidos por los sujetos, así como también los puntajes que le asignaron a los ítems de cada una de las tres dimensiones estudiadas, tanto antes como después de la aplicación del programa de orientación educativa. Se puede apreciar que no hubo uniformidad en las opiniones de

Tabla 1. Puntaje alcanzado por cada una de las dimensiones antes y después del programa de orientación educativa

\begin{tabular}{cccccc}
\hline \multirow{2}{*}{ Dimensiones } & \multicolumn{2}{c}{ Puntaje } & \multicolumn{2}{c}{ Prueba t student (t) } & Observaciones \\
\cline { 2 - 4 } & Antes & Después & t & Probabilidades & Iguales \\
\hline $\begin{array}{c}\text { Nivel de } \\
\text { conocimiento }\end{array}$ & 735 & 755 & $-1,25 \mathrm{NS}$ & $\mathrm{P}=0,2116$ & $\mathrm{P}=0,2850$ \\
\hline $\begin{array}{c}\text { Cuidados y medidas } \\
\text { preventivas }\end{array}$ & 832 & 850 & $-1,08 \mathrm{NS}$ & $\mathrm{P}=0,0017$ & Diferentes \\
\hline $\begin{array}{c}\text { Gestión de la salud } \\
\text { emocional }\end{array}$ & 700 & 765 & $-3,34 * *$ & & \\
\hline
\end{tabular}

NS: No significativo; *: Significativo $\mathrm{P}<0,05 ; * *$ : Altamente significativo $\mathrm{P}<0,01$ 
Tabla 2. Uniformidad en las opiniones y diferencias entre los puntajes en las tres dimensiones antes y después del programa de orientación

\begin{tabular}{|c|c|c|c|c|c|c|}
\hline \multirow{3}{*}{$\begin{array}{l}\text { Momento de la } \\
\text { medición }\end{array}$} & \multirow{2}{*}{\multicolumn{2}{|c|}{$\begin{array}{c}\text { Uniformidad entre las } \\
\text { opiniones en las tres } \\
\text { dimensiones }\end{array}$}} & \multicolumn{4}{|c|}{$\begin{array}{l}\text { Diferencias entre los puntajes asignados a las } \\
\text { dimensiones }\end{array}$} \\
\hline & & & \multicolumn{2}{|c|}{ Prueba Friedman (F) } & \multirow{2}{*}{ Dimensión } & \multirow{2}{*}{ Rango } \\
\hline & $\mathbf{F}$ & Probabilidades & $\mathbf{F}$ & probabilidades & & \\
\hline \multirow{3}{*}{$\begin{array}{l}\text { Antes del programa } \\
\text { de orientación }\end{array}$} & \multirow{3}{*}{75,152} & \multirow{3}{*}{$\mathrm{P}=0,0056^{* *}$} & \multirow{3}{*}{38,166} & \multirow{3}{*}{$\mathrm{P}=0,0000^{* * *}$} & 1 & 1,74 \\
\hline & & & & & 2 & 2,68 \\
\hline & & & & & 3 & 1,58 \\
\hline \multirow{3}{*}{$\begin{array}{l}\text { Después del } \\
\text { programa de } \\
\text { orientación }\end{array}$} & \multirow{3}{*}{52,927} & \multirow{3}{*}{$\mathrm{P}=0,2878 \mathrm{NS}$} & \multirow{3}{*}{28,777} & \multirow{3}{*}{$\mathrm{P}=0,0000^{* * *}$} & 1 & 1,66 \\
\hline & & & & & 2 & 2,59 \\
\hline & & & & & 3 & 1,75 \\
\hline
\end{tabular}

NS: No significativo; * : Significativo $\mathrm{P}<0,05 ; * *$ : Altamente significativo $\mathrm{P}<0,01 ; * * *$ : Muy altamente significativo $\mathrm{P}<0,001$

los sujetos antes del programa de orientación, mientras que sí hubo uniformidad después.

En cuanto a los puntajes asignados a los ítems de las dimensiones por parte de los sujetos, se encontraron diferencias muy altamente significativas tanto antes como después del programa, siendo la dimensión 2 (cuidados y medidas preventivas) la de mayor impacto en ambos casos, al mostrar el mayor rango (Tabla 2)

\section{DISCUSIÓN}

La convocatoria a participar en esta investigación se extendió a todos los residentes mayores de 18 años de la provincia de Chachapoyas; no obstante, los datos demográficos de la muestra indican que ésta estuvo constituida principalmente por adultos jóvenes con niveles de estudio de secundaria o universitaria completa. Con este perfil de los participantes sería de esperar que estas personas cuenten con conocimientos importantes sobre algunos aspectos esenciales de la pandemia, tales como sus causas, formas de transmisión, evolución de la enfermedad, así como también de los métodos de prevención y autocuidados correctos adquiridos en sus contextos académicos $y / u$ otras fuentes de información creíbles (Beltrán y Pérez, 2020; Sánchez et al., 2020). Este supuesto se confirmó en nuestro estudio en todas las tres dimensiones con puntajes altos o muy altos en la mayoría de los ítems, pero sobre todo en las dimensiones cuidados y medidas preventivas, y nivel de conocimiento sobre la enfermedad, las que alcanzaron las puntuaciones más altas antes de la aplicación del programa de orientación educativa (Tabla 1 ).
Si bien es cierto, el programa de orientación educativa produjo un incremento consistente de las puntuaciones en cada una de las tres dimensiones, este aumento sólo fue altamente significativo $(\mathrm{P}=0,0017)$ para la dimensión gestión de la salud emocional; justamente la dimensión en la que la puntuación antes del programa fue la más baja. Este resultado refuerza nuestra afirmación de que la población estudiada poseía un alto o muy alto nivel de conocimiento sobre la enfermedad y aplicaba las estrategias correctas para prevenirlas. Nuestros resultados coinciden con los de Beltrán y Pérez (2020) en Lima, Perú, quienes reportaron que los sujetos de edades entre 18 a 30 años y con educación técnica y universitaria mostraron niveles alto de conocimiento sobre la pandemia y sus causas, así como también los de Sánchez Mora et al. (2020) en México, de que los universitarios tenían una mayor comprensión de la transmisión de las enfermedades infecciosas, lo que les aportaba elementos para aplicar el pensamiento crítico en la toma de decisiones en cuanto a los comportamientos de autocuidado durante la pandemia, y los de Ruiz-Aquino et al. (2020) de que la población peruana joven y con educación universitaria tenia conductas más eficaces que el resto de la población en los autocuidados de la salud física y mental en la prevención de la COVID-19.

Los resultados de nuestra investigación sugieren que los participantes no solo tenían información correcta sobre la pandemia y que ponían en práctica las medidas preventivas adecuadas más comunes como el lavado frecuente de las manos, el uso de la mascarilla y 
no consumían medicamentos con fines preventivos, sino que también indican que las técnicas de tecnologías mentales transferidas, como las estrategias de afrontamiento centrada en el problema o las emociones, la meditación guiada, las técnicas de relajación mental y los cambios de creencias aplicados, tuvieron un efecto beneficioso muy altamente significativo en ellos. Se sabe que estas tecnologías mentales constituyen herramientas poderosas para controlar el estrés, modificar los pensamientos, así como también para cambiar las creencias y actitudes negativas y limitantes por otros más positivos, brindando a las personas una mejor gestión de su mundo emocional frente a las adversidades (Gallegos et al., 2020). El educar a otros sobre cómo mejorar el bienestar psicológico, es uno de los usos que los profesionales de la salud mental le pueden dar a las redes sociales, al ser ellos considerados por la gente como una fuente de información confiable (Tala y Vásquez, 2020).

Cuadra-Martínez et al. (2020) clasifican el impacto psicológico de una pandemia en las dimensiones emocional, cognitiva, conductual, salud mental, organizacional y psicosocial. En nuestra investigación encontramos que la percepción de riesgo de contraer la enfermedad y el programa de orientación incidieron positivamente en la experiencia emocional de los participantes, con niveles bajo de preocupación, temor, incertidumbre o síntomas de ansiedad por la posibilidad de enfermar tanto ellos como familiares cercanos. Algunos autores sugieren que la percepción de riesgo de enfermedad (Martin Alfonso et al., 2020), así como la intolerancia a la incertidumbre inducen a las personas a incrementar el monitoreo de su salud y que la ansiedad puede ser una respuesta adaptativa frente al peligro de contagio (Cabanillas-Rojas, 2020). Otro elemento digno de resaltar de esta investigación, es la consistencia en las diferencias estadísticamente significativas entre las dimensiones, en cuanto a los puntajes asignados por los sujetos a cada una de ellas, tanto antes como después del programa de orientación, siendo la dimensión 2 (cuidados y medidas preventivas) la que representó el mayor impacto, al proporcio- nar el mayor rango entre las dimensiones (Tabla 2). Este resultado sugiere que los participantes tuvieron comportamientos preventivos idóneos ante la enfermedad, durante el periodo de la investigación y, muy probablemente, mantuvieron dicho comportamiento en su vida cotidiana, después de finalizado el estudio. Cabe destacar que para la fecha del estudio había un aumento en la proporción de personas enfermas y fallecidas por COVID-19 en el país y en el departamento de Amazonas que afectó principalmente a los adultos mayores (Nieves-Cuervo et al., 2021; DIRESA, 2020; DGE, 2020).

Por otra parte, debe resaltarse que la cultura de los sujetos sobre la pandemia no era uniforme antes de la aplicación del programa, mientras que si lo fue después. Esta afirmación se evidencia en la diversidad de opiniones o juicios que emitieron sobre cada una de las dimensiones (Tabla 2). Estos resultados, junto con el incremento numérico de las puntuaciones logrado en cada una de las dimensiones después, sugieren que el programa aplicado promovió un aprendizaje equilibrado sobre la pandemia en los participantes. CatalánMatamoros (2020) sostiene que, para poder lograr una transferencia eficaz y eficiente de información sobre la pandemia por redes sociales, es necesario considerar el contexto sociocultural en el que se desarrolla, elaborar estrategias de comunicación que respondan a las necesidades de las audiencias, y tener en cuenta que los seres humanos no siempre seguimos un razonamiento lógico y racional. Cabe destacar también que las propias redes sociales se han dado cuenta de que la desinformación que por ellas se transmite es una amenaza para su estabilidad y sostenibilidad (LópezBorrull y Ollé, 2020).

En el diseño del programa de orientación educativa utilizado para esta investigación, se tomó en cuenta únicamente la información científica sobre la pandemia, validada y actualizada por las instituciones rectoras de la salud a nivel nacional y mundial, presentada en formato flexible y amena, donde se le daba la oportunidad a los sujetos para que retroalimentaran con sus aportes y opiniones sobre los contenidos trabajados y 
el nivel de comprensión logrado; todo con la finalidad de promover su participación en la búsqueda de conocimientos sobre el tema y generar un aprendizaje significativo y duradero. Se sabe que este enfoque educativo de aprender a aprender, permite mayor responsabilidad del participante, siendo concordante con el paradigma constructivista social del conocimiento, capaz de conducir hacia una educación sustentable (Cárdenas y Madero, 2020; Velez-Loor et al, 2020). Corresponde a los profesionales y especialistas sanitarios utilizar eficientemente las redes sociales para educar en salud a las poblaciones (Tala y Vásquez, 2020).

En nuestra investigación, solo se incluyó a la población urbana de la provincia estudiada, pues la población rural tanto local como nacional, que también es numerosa, presenta serias limitaciones de conectividad a Internet y con ello a las tecnologías de la información y comunicación, incluyendo las redes sociales (INEI, 2018; Gómez-Arteaga y Escobar-Mamani, 2021) haciendo difícil su participación en este estudio y en cualquier otro programa educativo que utilice estas plataformas para la educación virtual. Este marco plantea como problema central el acceso a la educación y el aprovechamiento de las tecnologías digitales para el logro de un sistema educativo más equitativo e igualitario, y pone a prueba las propuestas de inclusión digital educativa en la región (Lugo et al., 2020), así como también sus potencialidades para ayudar a mantener una cultura de paz en tiempos de COVID-19 (Navarro Rodríguez et al., 2020).

\section{CONCLUSIONES}

Nuestros hallazgos son coherentes con la perspectiva conceptual de las redes sociales como un recurso tecnológico valioso de la comunicación, capaz de llegar e impactar a las poblaciones, que si son aprovechadas de manera responsable y profesional para transferir e intercambiar contenidos científicos válidos y confiables sobre la pandemia COVID-19, es útil para ayudar a la gente a empoderarse de su propia salud y asumir conductas saludables y sustentables frente a la enfer- medad. Este rol protagónico de las redes sociales en la educación y la salud parece fortalecerse mientras persiste la emergencia sanitaria; sin embargo, para que puedan alcanzar un papel preponderante en la sustentabilidad educativa local y regional, es necesario que se superen las brechas y desigualdades de conectividad a Internet existente entre las poblaciones, muy particularmente entre las rurales y urbanas.

\section{VI.AGRADECIMIENTOS}

Los autores agradecen a las personas residentes en la provincia de Chachapoyas que voluntariamente aceptaron la invitación y dieron su consentimiento de participar, haciendo realidad esta investigación.

\section{CONTRIBUCIÓN DE LOS AUTORES}

Todos los autores participaron en la conceptualización, metodología, investigación, redacción del manuscrito inicial, revisión bibliográfica, y en la revisión y aprobación del manuscrito final.

\section{CONFLICTO DE INTERESES}

Los autores declaran no tener conflicto de intereses.

\section{REFERENCIAS BIBLIOGRÁFICAS}

Acosta-Quiroz, J, y S. Iglesias-Osore. 2020. “COVID19: Desinformación en redes sociales". Revista del cuerpo médico 13 (2). DOI:10.35434/rcmhnaaa.2020.132.678

Aguilar-Barojas, S. 2005. "Fórmulas para el cálculo de la muestra en investigaciones de salud". Saluden Tabasco 11 (1-2): 333-338.

Álvarez-Mon, M. A., A. Rodríguez-Quiroga, L. de Anta, y J. Quintero. 2020. “Aplicaciones médicas de las redes sociales. Aspectos específicos de la pandemia de la COVID-19”. Medicine - Programa de Formación Médica Continuada Acreditado. 13 (23): 1305-1310. DOI: 10.1016/j.med.2020.12.012

Beltrán Villanueva, K. R y I. G. Pérez Regalado. 2020. "Nivel De conocimiento sobre las medidas preventivas de COVID-19 en los pobladores 
de la urbanización Brisas de Santa Rosa III Etapa - San Martin de Porres”. Tesis de Grado. Universidad Nacional del Callao. Lima (Perú).

Bohórquez P, B., X. Luengo-Charath, M. V. Anguita, R. L. Bascuñán, I. M. Pacheco, C. P. Michaud, e Y. R. Vacarezza. 2020. "Uso y difusión responsable de la información en pandemia: un imperativo ético". Rev. chilena $\begin{array}{lllll}P \text { e d i a tr. } & 9 & 1 & \text { ( } 5 \text { ). } & \text { D O I : }\end{array}$ 10.32641/rchped.vi91i5.2420

Buitrago Ramírez, F., R. Ciurana Misol, M. C. Fernández Alonso, y J. L. Tizón. 2020. “Pandemia de la COVID-19 y salud mental: reflexiones iniciales desde la atención primaria de salud española”. Atención Primaria 53 (1): 89-101. DOI: 10.1016/j.aprim.2020.06.006 02126567

Cabanillas-Rojas, W. 2020. “Conducta y propagación del COVID-19 en el Perú: Marco de referencia para el diseño de intervenciones conductuales de salud pública”. DOI: 10.1590/SciELOPreprints.868

Cárdenas, P y C. Madero. 2020. Aprendizajes Profesionales en pandemia: Reflexiones de docentes, directivos y profesionales de la educación. Informe Técnico. Santiago (Chile).

Catalán-Matamoros. D. 2020. "La comunicación sobre la pandemia del COVID-19 en la era digital: manipulación informativa, fake news y redes sociales". Revista española de comunicación en salud. 1: S5-S8 DOI: $10.20318 /$ recs.2020.5531

Cuadra-Martínez, D., P. J. Castro-Carrasco, J. Sandoval-Díaz, D. Pérez-Zapata, y D. Mora Dabancens. 2020. "COVID-19 y comportamiento psicológico: revisión sistemática de los efectos psicológicos de las pandemias del siglo XXI". Rev. Med. Chile 148: 1139-1154

DGE (Dirección General de Epidemiología del Perú). 2020. Situación actual COVID-19. Informe Técnico. Lima (Perú).
DIRESA(Dirección Regional de Salud de Amazonas). 2020. Sala situacional COVID-19. Informe Técnico. Chachapoyas (Perú).

Gallegos, M., C. Zalaquett, S. E. Luna Sánchez, R. Mazo-Zea, B. Ortiz-Torres, J. C. PenagosCorzo, N. Portillo, I. Torres Fernández, A. Urzúak, M. Morgan Consoli, F. A. Polanco, A. M. Flórez, y R. Lopes Miranda. 2020. “Cómo afrontar la pandemia del coronavirus (COVID-19) en las Américas: Recomendaciones y líneas de acción sobre salud mental". Revista Interamericana de Psicologia/Interamerican Journal of Psychology 54 (1): e1304.

Gómez-Arteaga, I, y F. Escobar-Mamani. 2021. "Educación virtual en tiempos de pandemia: incremento de la desigualdad social en el Perú". Chakiñan 15 (12): 152-165.

Gonzales-Castillo, J. R., L. Varona-Castillo, M. G. Domínguez-Morante, y V. R. OcañaGutiérrez. 2020. "Pandemia de la coviD-19 y las Políticas de Salud Pública en el Perú: marzo-mayo 2020”. Rev. salud pública 22 (2): e387373.

Hao, K., y T. Basu. 2020. The coronavirus is the first true social-media "infodemic". https://www.technologyreview.com/2020/02 /12/844851/ the-coronavirus-is-the-first-truesocial-media-infodemic/ (Consultada el 28 de mayo de 2021)

INEI (Instituto Nacional de Estadística e Informática del Perú). 2017. Censos Nacionales 2017: XII de Población, VII de Vivienda y III de Comunidades Indígenas. Lima (Perú): INEI.

INEI (Instituto Nacional de Estadística e Informática del Perú). 2018. Perú: Línea de base de los principales indicadores disponibles de los objetivos de desarrollo sostenible (ODS). Lima (Perú): INEI.

Llerena Lanza, R. A, y C. A. Sánchez Narváez. 2020. "Emergencia, gestión, vulnerabilidad y respuestas frente al impacto de la pandemia 
COVID-19 en el Perú". DOI: DOI: 10.1590/SciELOPreprints.94

López-Borrull, A, y C. Ollé. 2020. “Curación de contenidos científicos en tiempos de fake science y Covid-19: una aproximación entre las ciencias de la información y la comunicación”. En VII Congreso Internacional de la Asociación Española de Investigación de la Comunicación (AE-IC) Valencia (España). DOI: 10.3145/AE-IC-epi.2020.e16

Lugo, M. T., V. Ithurburu, A. Sonsino, y F. Loiacono. 2020. "Políticas digitales en educación en tiempos de Pandemia: desigualdades y oportunidades para América Latina”. EDUTEC. Revista Electrónica de Tecnología Educativa $73: \quad 23-36 . \quad$ D O I : 10.21556/edutec.2020.73.1719.

Martín Alfonso, L., L. Martínez Debs, A. C. CencioBello, M. Rodríguez Martínez, y J. Ortiz Gómez. 2020. "Percepción de riesgo, búsqueda de ayuda médica y autocuidado: Retos psicológicos frente a la COVID-19". Revista cubana de psicología 2 (2): 2-17

Nieves-Cuervo, G. M., E. F. Manrique-Hernández, A. F. Robledo-Colonia, y E. K. Grillo Ardila. 2021. "Infodemia: Noticias falsas y tendencias de mortalidad por COVID.19 en seis países de América Latina". Rev. Panam. Salud Publica 45: e44. doi: 10.26633/RPSP.2021.44

Navarro Rodríguez, J. D., O. Medina-Ortiz, C. Andrade Fossi, M. Chacín González, y Bermúdez, V. 2020. "Cultura de paz en la pandemia por SARS-COV-2: confinamiento, tics y las redes sociales". Gac Méd Caracas 128 (2): S350S358. DOI: 10.47307/GMC.2020.128.s2.21

Rubio Hurtado, M. J. y Berlanga Silvente, V. 2012. "Cómo aplicar las pruebas paramétricas bivariadas $\mathrm{t}$ de Student y ANOVA en SPSS. Caso práctico". REIRE, Revista d'Innovació $i$ Recerca en Educació 5 (2): 83-100.

Ruiz-Aquino, M., A. Díaz-Lazo, M. L. Ortiz-Aguí, y E. I. Villar Carbajal. 2020. "Conductas de autocuidado en la prevención del contagio de la COVID-19 en pobladores peruanos". Revista Cubana de Medicina General Integral36(4): e1708.

Salaverria, R., N. Buslón, F. López-Pan, B. León, I. López-Goñi, y M. C. Erviti. 2020. "Desinformación en tiempos de pandemia: tipología de los bulos sobre la Covid-19". El profesional de la información 29 (3): e290315. DOI: 10.3145/epi.2020.may.15

Sánchez-Mora, M. C., P. Aguilera-Jiménez, M. Y. Hernández-Arellano, y M. L Patiño-Barba. 2020. "Aplicación de los conocimientos científicos y la prevención de COVID-19 en México en tiempos de posverdad". Revista Prisma Social 31 (4): 82-109

Sandín, B., R.M. Valiente, J. García-Escalera, y P. Choro. 2020. "Impacto psicológico de la pandemia de COVID-19: Efectos negativos y positivos en población española asociados al periodo de confinamiento nacional”. Journal of Psychopathology and Clinical Psychology/Revista de Psicopatología y Psicología Clinica 25 (1): 1-22. D O I : 10.5944/rppc.27569

Serri, M. 2018. "Redes sociales y Salud". Rev Chilena Infectol 35 (6): 629-630

Tala, A, y E. Vásquez. 2020. “Conexión en tiempos de COVID-19”. Rev Med Chile. 148: 557-564.

Vélez-Loor, M. D., P. A. Vallejo-Valdivieso, y M. E. Moya-Martínez. 2020. "Recursos didácticos virtuales en proyectos de ciencias naturales en período de confinamiento por COVID-19”. Episteme Koinonia. 3 (5): 183-201. DOI: 10.35381/e.k.v3i5.760. 